\title{
Triptolide exerts protective effects against fibrosis following ileocolonic anastomosis by mechanisms involving the miR-16-1/HSP70 pathway in IL-10-deficient mice
}

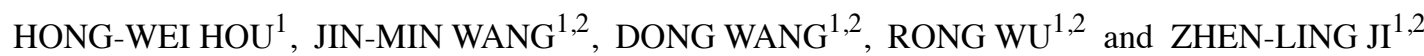 \\ ${ }^{1}$ Department of General Surgery, Southeast University Medical School; \\ ${ }^{2}$ Zhongda Hospital, Southeast University, Nanjing, Jiangsu 210009, P.R. China
}

Received January 31, 2017; Accepted May 31, 2017

DOI: $10.3892 /$ ijmm.2017.3016

\begin{abstract}
Surgeries, particularly ileocecal resection (ICR), are often required in the treatment of Crohn's disease (CD). However, recurrences are common for patients who undergo ICR, and anastomotic fibrosis is the main cause of re-operation. The present study aimed to investigate the therapeutic effects of triptolide (TPL) in ameliorating fibrosis following ileocolonic anastomosis. A model of IL-10 $1 /-$ mice undergoing ICR was used to study postsurgical inflammation and fibrosis of anastomosis. For this purpsose, interleukin (IL)- $10^{-/-}$mice were randomly divided into 3 groups as follows: the control group, the saline-treated group subjected to ICR (ST-ICR) and the TPL-treated group subjected to ICR (TT-ICR). Wild-type (WT) mice of matching ages were assigned to the WT group. The effects of TPL treatment on ileocolonic anastomosis were determined by histopathologicalevaluation, westernblotanalysis and ELISA. The analysis of the effects of TPL treatment on microRNA-16-1 (miR-16-1) and heat shock protein 70 (HSP70) expression was carried out by RT-qPCR and western blot analysis. Compared with the control group, significantly higher inflammation scores following anastomosis were observed in the ST-ICR group $(\mathrm{P}<0.05)$, although reversion was observed in the TT-ICR group, which was consistent with changes in the area of $\mathrm{CD}_{4}{ }^{+}$cell infiltration. The elevated fibrosis scores and the overexpression of procollagen I and III in the ST-ICR group were all inhibited by TPL. With an increase in the severity of inflammation and fibrosis, the levels of IL-6, tumor necrosis factor- $\alpha(\mathrm{TNF}-\alpha)$ and transforming growth factor- $\beta 1$ (TGF- $\beta 1)$ increased; however, a significant decrease in these levels was
\end{abstract}

Correspondence to: Dr Zhen-Ling Ji or Dr Rong Wu, Department of General Surgery, Southeast University Medical School, 87 Ding Jia Qiao Road, Nanjing, Jiangsu 210009, P.R. China

E-mail: zlji@vip.sina.com

E-mail:wrwr615615@sina.com

Key words: triptolide, interleukin-10 deficient mice, ileocecal resection, Crohn's disease, fibrosis, miR-16-1/heat shock protein 70 pathway observed following treatment with TPL $(\mathrm{P}<0.05)$. The results of RT-qPCR revealed that the upregulated miR-16-1 levels in the ST-ICR group were significantly reduced by TPL. HSP70, which can be inhibited by miR-16-1, ameliorates anastomotic inflammation and fibrosis. Thus, the present study demonstrates that TPL exerts a protective effect against fibrosis following anastomosis in CD. The miR-16-1/HSP70 signaling pathway, which can be regulated by TPL, may thus represent a novel therapeutic option in $\mathrm{CD}$ that deserves further investigation.

\section{Introduction}

Inflammatory bowel disease (IBD) is a group of complex intestinal disorders that includes ulcerative colitis (UC) and Crohn's disease (CD), both of which are characterized by chronic inflammation and damage, and the destruction of tissue architecture. Overall morbidity is relatively high in developed countries, and an increasing prevalence in the developing world has been noted, particularly for CD (1). In contrast to $\mathrm{UC}, \mathrm{CD}$ is characterized by patchy, transmural inflammation, which may lead to perforation or stricture of the digestive tract. $\mathrm{CD}$ is defined by age at onset, and the location and pattern of disease, which are combined in the Vienna classification (2). Genetic and environmental factors all play important roles in $\mathrm{CD}$, although a definitive pathogenesis remains unknown.

Intestinal fibrosis, defined as an excessive synthesis of connective tissue in the intestinal wall, is a common complication of $\mathrm{CD}$ that is associated with long-term enervating consequences that impair the quality of life of patients. Although surgical treatment is not curative for $\mathrm{CD}$, it is still required for approximately $70 \%$ of $\mathrm{CD}$ patients if medical treatments are ineffective or to ameliorate complications, such as obstruction caused by intestinal fibrosis. One of the key points for reducing the rate of re-operation is to alleviate fibrosis at the site of anastomosis.

The primary therapy for CD is pharmaceutical-based, which is evolving rapidly with many new biological agents under investigation. Possessing both immunomodulatory and anti-inflammatory effects, triptolide (TPL), an active component of Tripterygium wilfordii Hook F., has been used as a therapeutic agent for a number of autoimmune diseases, including $\mathrm{CD}$, for several years. A previous study showed that 
extracts of Tripterygium wilfordii Hook F. exhibited therapeutic activity in mild or moderately active $\mathrm{CD}(3)$. Many mechanisms of TPL have been reported in previous studies, including the inhibition of the expression of XPB (a subunit of TFIIH) (4), the Toll-like receptor (TLR)/nuclear factor (NF)- $\mathrm{BB}$ signaling pathway (5) and the tumor necrosis factor (TNF)- $\alpha$ /TNFR2 signaling pathway (6). Recently, a novel model involving interleukin (IL)-10 $10^{--}$mice undergoing ICR has provided a novel method with which to investigate fibrosis following anastomosis in $\mathrm{CD}(7)$.

TPL was used as a therapeutic substance in this new model of IL-10 $0^{-/}$mice undergoing ICR in the present study. The aim of this study was to investigate the therapeutic effects of TPL in ameliorating fibrosis following anastomosis.

\section{Materials and methods}

Animals and surgical procedure. Male $\mathrm{C} 3 \mathrm{H} / \mathrm{HeJBir} \mathrm{IL}-10^{-1-}$ and wild-type (WT) mice ( 8 weeks old) were purchased from Jackson Laboratory (Ben Harbor, ME, USA) and raised under specific pathogen-free (SPF) conditions at the Medical School of Southeast University, Nanjing, China. A total of $18 \mathrm{IL}^{-10^{-/-}}$mice were evenly and randomly divided into 3 groups as follows: the control group (no intervention), the ST-ICR group (ICR and intraperitoneally injected saline) and the TT-ICR group (ICR and intraperitoneally injected TPL). A total of 6 WT mice of matched ages were assigned to the WT group (no intervention). Mice were fasted 1 day before the beginning of the experiment. Surgeries were performed under sterile conditions with the assistance of a surgical microscope (x7 magnification). An intestinal segment comprising of $5 \mathrm{~cm}$ of small bowel proximal to the ileocecal junction and $2 \mathrm{~cm}$ distal to the proximal colon was resected after ligating the mesentery. The intestinal continuity was restored with a single-layered, interrupted, end-to-end anastomosis with a 9-0 monofilament suture. Animals surviving less than 4 days after surgery were excluded. All protocols for this animal research were approved by the Ethics Committee of Southeast University.

Administration protocol of drugs and assessment of disease activity. TPL (chemical structure shown in Fig. 1; Solarbio, Beijing, China) with a purity $\geq 99 \%$ was dissolved in dimethyl sulfoxide (DMSO; Solarbio) at a concentration of $0.0035 \mathrm{mg} /$ $\mathrm{ml}$. A $0.07 \mathrm{mg} / \mathrm{kg}$ body weight dose of TPL was selected as the appropriate dose to inject mice intraperitoneally in the TT-ICR group every other day for 8 consecutive weeks, as previously described (6). Mice in the ST-ICR group were administered equal amounts of saline.

A disease activity index (DAI) was employed to evaluate the status of the mice after undergoing ICR for 8 weeks. Three parameters were taken into account as previously described: weight loss, stool consistency and bleeding (8). Each clinical parameter was assigned a value ranging from $0-4$ (with 4 being the most severe). The final value of the DAI was calculated as (the sum of the scores for weight loss, stool consistency and bleeding) $/ 3$.

Tissue collection. A total of 8 weeks after the beginning of the experiment, the mice were weighed and sacrificed by decapitation. A $1 \mathrm{~cm}$ segment of the small intestine (SI) spanning the

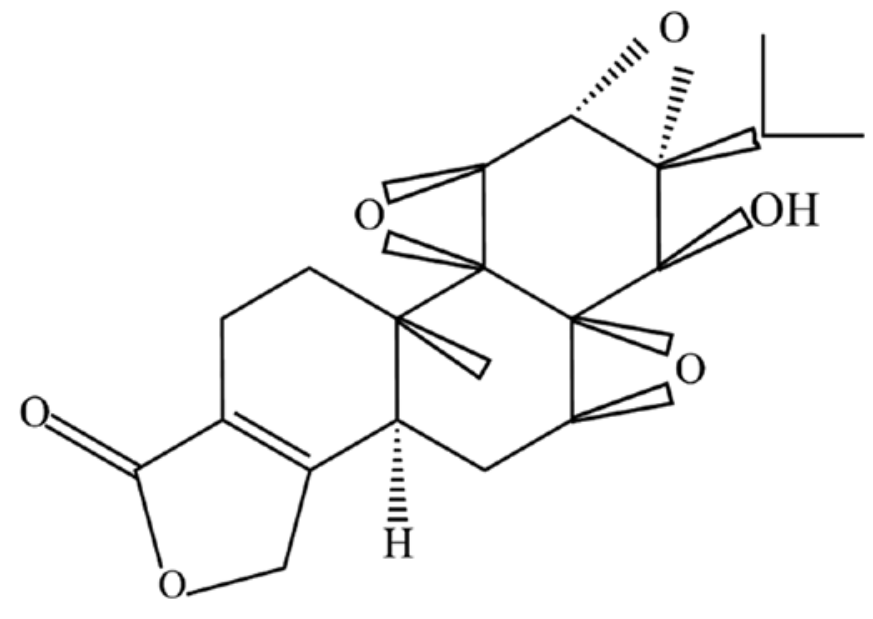

Figure 1. Structure of triptolide (TPL).

ileocecal junction in the WT and control groups and a $1 \mathrm{~cm}$ segment of the SI proximal to the anastomosis in the ICR groups was collected for histological and immunohistochemical analyses, reverse transcription-quantitative PCR (RT-qPCR), ELISA and western blot analysis.

Histological analysis. The samples were fixed in $10 \%$ neutral-buffered formalin for $24 \mathrm{~h}$ and then embedded in paraffin and sectioned at a thickness of $5 \mu \mathrm{m}$. Xylene was used to remove the paraffin; the slides were then cleared with alcohol and subjected to $\mathrm{H} \& \mathrm{E}$ (hematoxylin and eosin) and Masson's trichrome staining (both from Servicebio, Wuhan, China). The evaluation of histological inflammation scores (0-4, with 4 being the most severe inflammation) based on H\&E staining was performed in a blinded manner according to previously described criteria $(9,10)$. This well-validated grading system is based on the severity and extent of leukocyte infiltration, epithelial hyperplasia, architectural distortion and depletion of goblet cells.

Masson's trichrome staining was used to visualize collagen accumulation. Fibrosis scoring was conducted based on the extent of collagen deposition on a scale from $0-3$, with 0 representing no collagen deposition. The details of the grading criteria were designed as follows: 0 , no significant collagen deposition; 1 , increased collagen deposition in submucosa and mucosa; 2 , increased collagen deposition in submucosa, mucosa and muscular layer; 3 , increased collagen deposition throughout all layers, including serosa. All scoring was performed by a single qualified pathologist who was blinded to the intervention.

Western blot analysis. Protein was extracted from the SI tissue using lysis buffer $(150 \mathrm{mM} \mathrm{NaCl} ; 0.5 \%$ sodium deoxycholate; $50 \mathrm{mM}$ Tris, $\mathrm{pH}$ 8.0, containing 1.0\% NP-40; $1.5 \mathrm{mM}$ EDTA; $10 \%$ glycerol) supplemented with a protease inhibitor cocktail (Bioss, Beijing, China) on ice. Supernatants were collected and centrifuged at $15,000 \mathrm{x} \mathrm{g}$ for $20 \mathrm{~min}$ at $4^{\circ} \mathrm{C}$. The protein concentration was measured using a $\mathrm{BCA}$ protein assay kit (Bioss). A total of $20 \mu \mathrm{g}$ of total protein was resolved by $10 \%$ sodium dodecylsulfate-polyacrylamide gel electrophoresis (SDS-PAGE) and transferred to polyvinylidene difluoride (PVDF) membranes by electroblotting at $4^{\circ} \mathrm{C}$. After 
Table I: Primer sequences for PCR.

\begin{tabular}{lll}
\hline Gene & \multicolumn{1}{c}{ Forward primer } & \multicolumn{1}{c}{ Reverse primer } \\
\hline miR-16-1 & 5'-CCGCTCGAGTGCAGGCCATATTGTGCTGCC-3' & 5'-TCCCCGCGGATTGTCTTCTAAGCTCTGTTC-3' \\
18S RNA & 5'-GTAACCCGTTGAACCCCATT-3' & 5'-CCATCCAATCGGTAGTAGCG-3' \\
HSP70 & 5'-GGCTGATCGGCCGCAAGTT-3' & 5'-AACTGCACCCACTTCCCAGTC-3' \\
$\beta$-actin & 5'-ACCACAGCTGAGAGGGAAATCG-3' & 5'-AGAGGTCTTTACGGATGTCAACG-3' \\
\hline
\end{tabular}

HSP70, heat shock protein 70 .

blocking with $5 \%$ skim milk for $2 \mathrm{~h}$ at room temperature, the membranes were incubated with primary antibodies overnight at $4^{\circ} \mathrm{C}$. Following washing with TBST 3 times, the membranes were then incubated with HRP-conjugated secondary antibody (Cat. no. bs-0369M-HRP, Bioss) at a dilution rate of 1:10,000 for $1 \mathrm{~h}$ at room temperature. After washing with TBST 3 times, the membranes were then incubated with ECL (Amresco, LLC, Solon, OH, USA) and analyzed with a FluorChem FC system (Alpha Innotech, San Leandro, CA, USA). The levels of protein expression were normalized to those of $\beta$-actin. The primary antibodies used were as follows: rabbit anti-mouse procollagen-I (Cat. no. ab64409; Abcam, Cambridge, UK), procollagen-III (GTX39505; Genetex, Irvine, CA, USA) and heat shock protein 70 (HSP70; Cat. no. ab79852; Abcam) monoclonal antibodies. All primary antibodies were used at a dilution of 1:200.

Immunohistochemistry. Slides were prepared as described above in the 'Histological analysis' section. Following incubation with $3 \% \mathrm{H}_{2} \mathrm{O}_{2}$ and $10 \%$ methanol in $0.01 \mathrm{M}$ PBS for $15 \mathrm{~min}$, the sections were blocked with $10 \%$ normal goat serum in $0.01 \mathrm{M}$ PBS for $1 \mathrm{~h}$ and incubated at $4^{\circ} \mathrm{C}$ overnight with

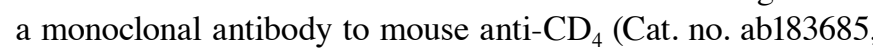
Abcam) diluted 1:100 with 5\% normal goat serum in Tris-Triton buffer. The sections were then incubated at room temperature for $1 \mathrm{~h}$ with goat anti-mouse (Cat. no. 115-505-003; Jackson Laboratory, Ben Harbor, ME, USA) 1:500 with Tris-Triton buffer. Finally, sections were visualized using the avidin-biotin DAB kit from Vector Laboratories, Inc. (Burlingame, CA, USA). Slice images (magnification, $x 100$ ) were captured with a digital camera (Leica, Mannheim, Germany), which was linked to a microscope and managed with OPTIMAS ${ }^{\mathrm{TM}}$ software version 6.1 (Optima Corp., Doral, FL, USA). The analyses of the number of cells per area $\left(12,234 \mu \mathrm{m}^{2}\right)$ were performed in 3 random areas.

RNA isolation and RT-qPCR. Total intestinal tissue RNA was isolated using the TRIzol ${ }^{\circledR}$ Plus RNA Purification kit (Invitrogen, Carlsbad, CA, USA) according to the manufacturer's instructions. 18S RNA was used as an internal control. qPCR was performed using the SYBR ${ }^{\circledR}$ Premix Ex Taq ${ }^{\mathrm{TM}}$ II PCR kit (Takara Bio, Inc., Otsu, Japan).

A total of $4 \mathrm{mg}$ of DNase-treated (Ambion, Austin, TX, USA) RNA was reverse transcribed into cDNA using oligo (dT) primers and reverse transcriptase (Promega Corp., Madison, WI, USA) under standard conditions. $\beta$-actin served as a control for HSP70. RT-PCR was performed using the StepOne ${ }^{\mathrm{TM}}$ and
StepOnePlus $^{\mathrm{TM}}$ Real-Time PCR System (Applied Biosystems Life Technologies, Foster City, CA, USA). Cycle thresholds for each test mRNA were recorded and normalized to a control. The denaturation process occurred at $95^{\circ} \mathrm{C}$ for $2 \mathrm{~min}$, and then PCR amplification was performed with 40 cycles at $95^{\circ} \mathrm{C}$ for $15 \mathrm{sec}$ and annealing at $60^{\circ} \mathrm{C}$ for $1 \mathrm{~min}$. The relative expression of microRNA/miRNA-16-1 (miR-16-1 or HSP70 was measured using the $\Delta \Delta \mathrm{Cq}$ method. The abundance of miR-16-1 or HSP70 mRNA was presented as the fold change from the mean expression levels in WT mice. The sequences of the primers are listed in Table I.

Cytokine-specific ELISA. Protein was extracted from the intestinal tissue by homogenization $(0.5 \mathrm{mg}$ tissue $/ \mathrm{ml})$ in homogenization buffer with a protease inhibitor (Bioss). Homogenized tissue samples were collected following centrifugation at $20,000 \mathrm{xg}$ at $4^{\circ} \mathrm{C}$ for $30 \mathrm{~min}$, and the supernatants were then stored at $-80^{\circ} \mathrm{C}$. The concentrations of TGF- $\beta 1, \mathrm{TNF}-\alpha$ and IL-6 were evaluated using a kit from Abcam according to the manufacturer's instructions. Values were expressed as pg/ mg protein.

Statistical analysis. Statistical significance among groups were calculated with one-way ANOVA testing: parametric data were assessed with a Bonferroni post hoc test and non-parametric data were assessed with the Kruskal-Wallis test with the Dunn's post hoc test (SPSS 21.0; IBM SPSS, Armonk, NY, USA). The data are expressed as the means \pm standard deviation (SD). A value of $\mathrm{P}<0.05$ was considered to indicate a statistically significant difference.

\section{Results}

TPL alleviates clinical symptoms and intestinal macroscopic changes in mice subjected to anastomosis. The IL-10 ${ }^{-/}$mouse represents a well-established animal model of $\mathrm{CD}$ owing to its persistent and dominant pro-inflammatory systemic immune profile. The model using IL-10 ${ }^{-/}$mice undergoing ICR was adopted in our study to investigate post-operative anastomotic inflammation and fibrosis in CD.

The mice in the ST-ICR group exhibited progressive weight loss, severe diarrhea, bowel wall edema and thickening, whereas no obvious signs of such symptoms were observed in the WT and control groups. In the mice in the TT-ICR group, slight diarrhea and weight loss were observed shortly after surgery, while bowel function recovered promptly following treatment with TPL, and no serious complications were observed in the 


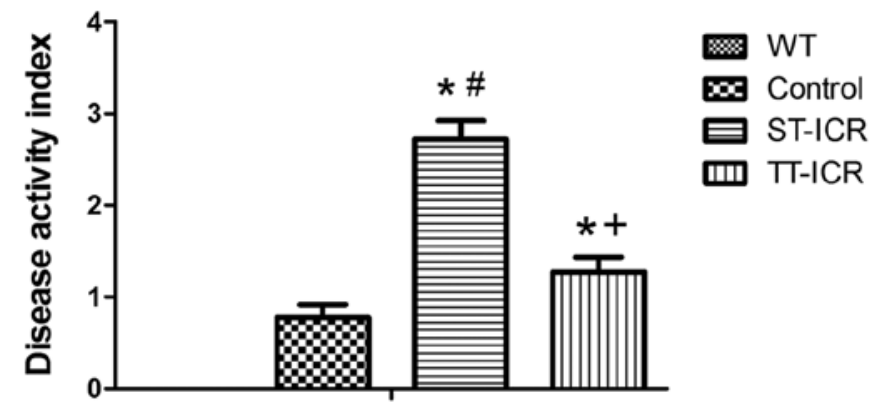

Figure 2. DAI scores for each group were assessed 8 weeks after surgery. Data are presented as the average \pm SD of 6 independent experiments. ${ }^{*} \mathrm{P}<0.05$, significantly different from the WT group; ${ }^{*} \mathrm{P}<0.05$, significantly different from the control group; ${ }^{+} \mathrm{P}<0.05$, significantly different from the ST-ICR. DAI, disease activity index; ICR, ileocecal resection; WT, wild-type; ST-ICR, saline-treated-ICR group; TT-ICR, triptolide-treated ICR group.

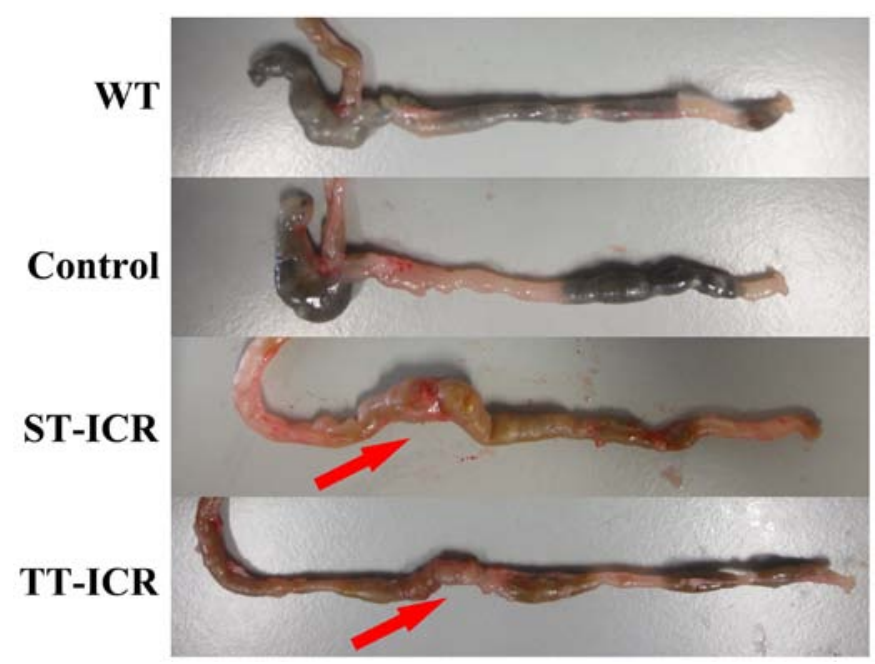

Figure 3. Photograph of macroscopic view of the intestinal tract involving the ileocecal junction. Anastomosis sites are indicated by red arrows. ICR, ileocecal resection; WT, wild-type; ST-ICR, saline-treated-ICR group; TT-ICR, triptolide-treated ICR group.

mice who survived $>2$ weeks after surgery. The DAI score of each group at the 8th week after surgery is shown in Fig. 2. No significant differences were observed between the WT and control groups $(\mathrm{P}>0.05)$. The DAI score in the ST-ICR group was significantly higher than that in the control group $(\mathrm{P}<0.05)$; however, a reduction was observed following treatment with TPL $(\mathrm{P}<0.05)$.

The bowels of the mice in the WT and control groups showed no macroscopic signs of inflammation, whereas the intestinal tract and, in particular, the anastomosis site of the mice in the ST-ICR group removed 8 weeks after surgery revealed evident hyperemia and inflammation (Fig. 3). Specifically, significant anastomotic lumen stenosis was observed in the mice in the ST-ICR group, while ICR-induced intestinal inflammation and anastomotic stenosis were effectively attenuated by TPL, although intestinal walls immediately adjacent to the anastomosis site still exhibited mild thickening in the mice in the TT-ICR group.

TPL attenuates post-surgical inflammation and anastomotic fibrosis in IL-10 $10^{-/}$mice that underwent ICR. H\&E staining and inflammation scores were employed to determine the severity of anastomotic inflammation. No obvious inflammation or destruction of the tissue architecture was observed in the WT or control groups. A total of 8 weeks after undergoing ICR, significant anastomotic microvilli swelling, epithelial hyperplasia, and architectural distortion accompanied by leukocytic infiltration were observed in the ST-ICR group compared to the control group, and all of these pathological abnormalities were significantly attenuated by treatment with TPL (Fig. 4A). The inflammation score, based on H\&E staining of the anastomosis site, was further investigated in IL-10 ${ }^{-/-}$mice (Fig. 4B). Compared with the control group, significantly higher inflammation scores of the anastomosis site were observed in the ST-ICR group $(\mathrm{P}<0.05)$, and a reduction was observed after following treatment with TPL $(\mathrm{P}<0.05)$.

In patients with $\mathrm{CD}$, over-activated Th1/Th17 type $\mathrm{T}$ cells are the main resources that induce local inflammatory reactions by producing pro-inflammatory cytokines; therefore, as a specific marker of $\mathrm{Th}$ cells, $\mathrm{CD}_{4}$ was examined by immunohistochemistry (Fig. 4A). The area of $\mathrm{CD}_{4}{ }^{+}$cell infiltration in the anastomosis site of the ST-ICR group was significantly larger than that in the WT and control groups $(\mathrm{P}<0.05$; Fig. $4 \mathrm{C})$, and $\mathrm{CD}_{4}{ }^{+}$cells were found infiltrating throughout the entire intestinal wall, particularly in the submucosa and mucosa. By contrast, following treatment with TPL, the $\mathrm{CD}_{4}{ }^{+}$area was diminished and showed no significant difference compared with the control group $(\mathrm{P}>0.05)$.

Collagen, which is the main component of the extracellular matrix (ECM), is mainly synthesized by fibrotic cells and indicates the severity of fibrosis (11). In this study, to determine whether intestinal fibrosis was the cause of luminal stenosis, the accumulation of collagen was visualized in the anastomosis site with Masson's staining 8 weeks after performing ICR (Fig. 5). No obvious deposition of collagen was observed in the WT and control groups, whereas significant collagen deposition throughout all layers of the intestinal wall was observed in the ST-ICR group compared to the WT and control groups. Once collagen synthesis becomes uncontrollable, thickening and stiffness of the bowel wall and luminal stenosis may be present; however, TPL treatment effectively attenuated the excessive accumulation of collagen. Furthermore, compared with the control group, the fibrosis scores in the ST-ICR group were significantly increased $(\mathrm{P}<0.05)$, whereas a significant reduction was observed in the TT-ICR group $(\mathrm{P}<0.05$; Fig. $6 \mathrm{~A})$.

Collagen molecules are synthesized from larger precursor proteins, known as procollagens. Thus, the synthesis rate of fibrillar collagens was further determined by measuring procollagen I and III by western blot analysis. As shown in Fig. 6B-D, no noteworthy differences were detected between the WT and control groups $(\mathrm{P}>0.05)$. Compared with the WT and control groups, the upregulated levels of procollagen I and III in the ST-ICR group were significantly decreased by TPL treatment $(\mathrm{P}<0.05)$. These results indicate that the balance between the synthesis and catabolism of ECM was altered in the IL-10 ${ }^{-/}$mice that underwent ICR.

TPL inhibits the secretion of inflammatory and fibrotic cytokines. IL-6, TNF- $\alpha$ and TGF- $\beta 1$ are efficient risk indicators and are closely related to the disease activity of CD. TGF- $\beta 1$, the most potent activator of fibroblasts, is crucial for the progres- 
A

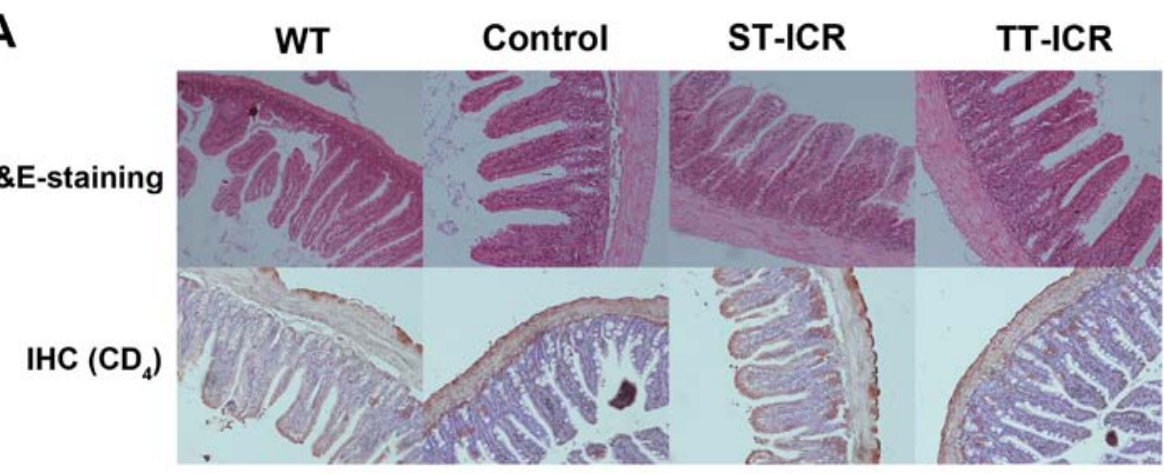

B

C
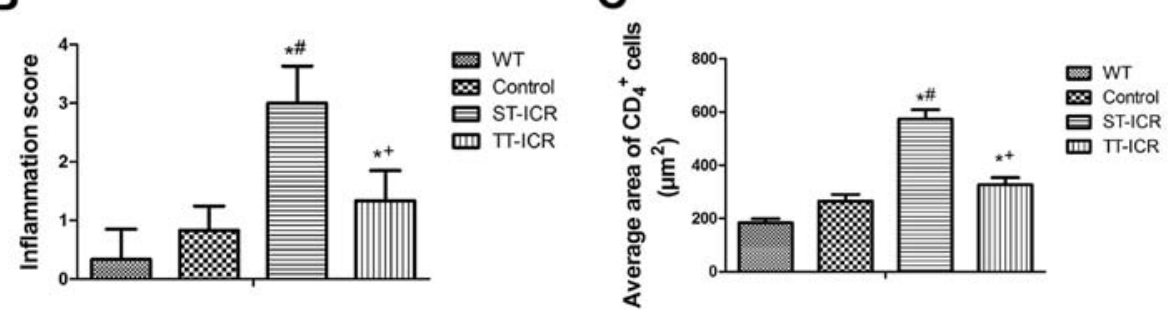

Figure 4. $\mathrm{H} \& \mathrm{E}$ staining and $\mathrm{IHC}$ of $\mathrm{CD}_{4}$ were employed to evaluate the severity of inflammation at the site of anastomosis in IL-10 ${ }^{-/}$mice that underwent ICR. (A) Histopathological changes at the site of anastomosis in each group. H\&E staining was performed to visualize the degree of inflammation at the site of anastomosis (magnification, $\mathrm{x} 100$ ); IHC was used to demonstrate the infiltration of $\mathrm{CD}_{4}{ }^{+}$cells (magnification, $\mathrm{x} 100$ ). (B) Anastomotic inflammation score of each group; (C) result of IHC for number of $\mathrm{CD}_{4}{ }^{+}$cells evaluated by image analysis (total area per field $=12,234 \mu \mathrm{m}^{2}$ ). The data are presented as the average $\pm \mathrm{SD}$ of 6 independent experiments. ${ }^{*} \mathrm{P}<0.05$, significantly different from the WT group; ${ }^{*} \mathrm{P}<0.05$, significantly different from the control group; ${ }^{+} \mathrm{P}<0.05$, significantly different from the ST-ICR group. IHC, immunohistochemistry; ICR, ileocecal resection; WT, wild-type; ST-ICR, saline-treated-ICR group; TT-ICR, triptolidetreated ICR group.
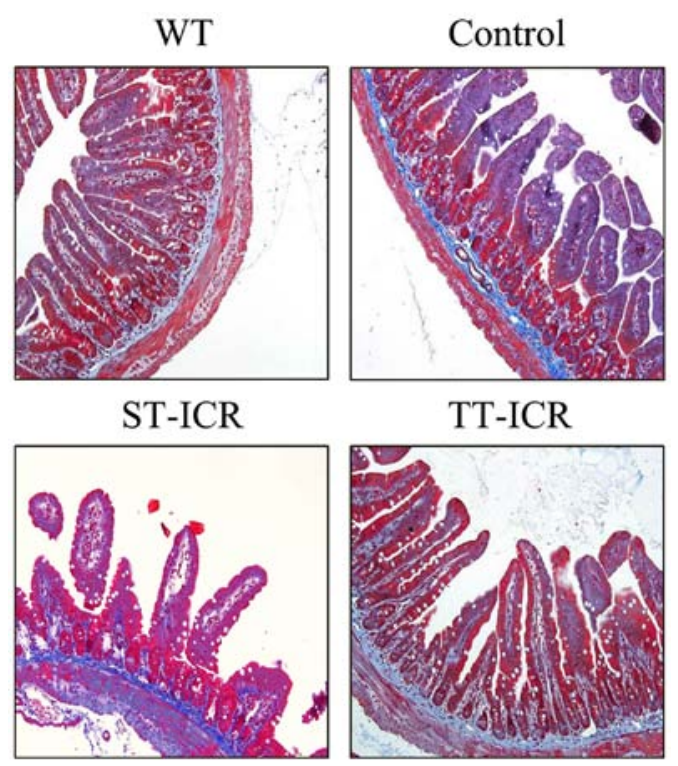

Figure 5. TPL ameliorates the fibrosis of anastomosis. Masson's trichrome stain of the anastomosis in each group was used to demonstrate the accumulated collagen deposition (stained blue) (magnification, x100). TPL, triptolide. ICR, ileocecal resection; WT, wild-type; ST-ICR, saline-treated-ICR group; TT-ICR, triptolide-treated ICR group.

sion of fibrosis (12-14). In this study, to further verify the degree and pathogenesis of inflammation and anastomotic fibrosis in the IL-10 ${ }^{-/}$mice that underwent ICR, cytokine-specific ELISAs were utilized to investigate the expression of IL-6, TNF- $\alpha$ and TGF- $\beta 1$ (Fig. 7). With the increasing severity of inflammation and fibrosis, the anastomotic concentrations of TGF- $\beta 1$, IL-6 and TNF- $\alpha$ all increased. Following treatment with TPL, these levels were all significantly decreased $(\mathrm{P}<0.05)$. Of note, although no significant difference in inflammation and fibrosis was detected between the WT and control groups in macroscopic or histopathologic views, the levels of IL-6 and TNF- $\alpha$ in the control group were significantly higher than those in the WT group $(\mathrm{P}<0.05)$.

TPL attenuates ileocolonic anastomotic fibrosis by regulating the miR-16-1/HSP70 pathway. As miR-16-1 is closely associated with the activity of CD $(15,16)$ and can be regulated by TPL (17); the level of miR-16-1 was further investigated by RT-qPCR (Fig. 8A). The level of miR-16-1 in the ST-ICR group was significantly elevated compared with the WT group $(\mathrm{P}<0.05)$. Compared with the control group, a higher level of miR-16-1 at the anastomosis site was observed in the ST-ICR group $(\mathrm{P}<0.05)$, although this increase was reversed by TPL $(\mathrm{P}<0.05)$. Therefore, we demonstrated that the expression of miR-16-1 can be effectively inhibited by TPL, and the DAI score can be reduced through the inhibition of miR-16-1.

As a main member of the HSP family, HSP70 can inhibit inflammation and fibrosis through mechanisms, such as the suppression of the expression of cytokines, regulating myofibroblast and $\mathrm{T}$ cell differentiation, and the inhibition of epithelial cell apoptosis $(18,19)$. More importantly, miR-16-1 targets the $3^{\prime}$ untranslated region (3'UTR) of HSP70 and reduces HSP70 expression (20). Therefore, in this study, RT-qPCR and western blot analysis were used to investigate the level of HSP70 and verify the association between HSP70 and 


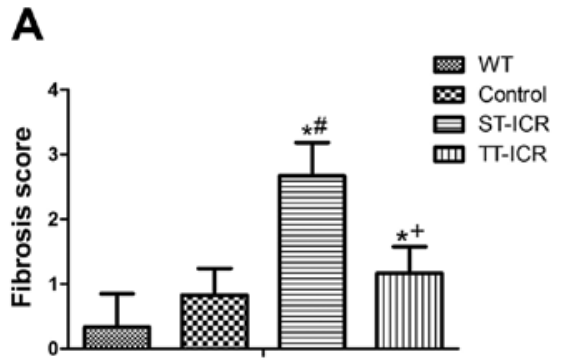

B
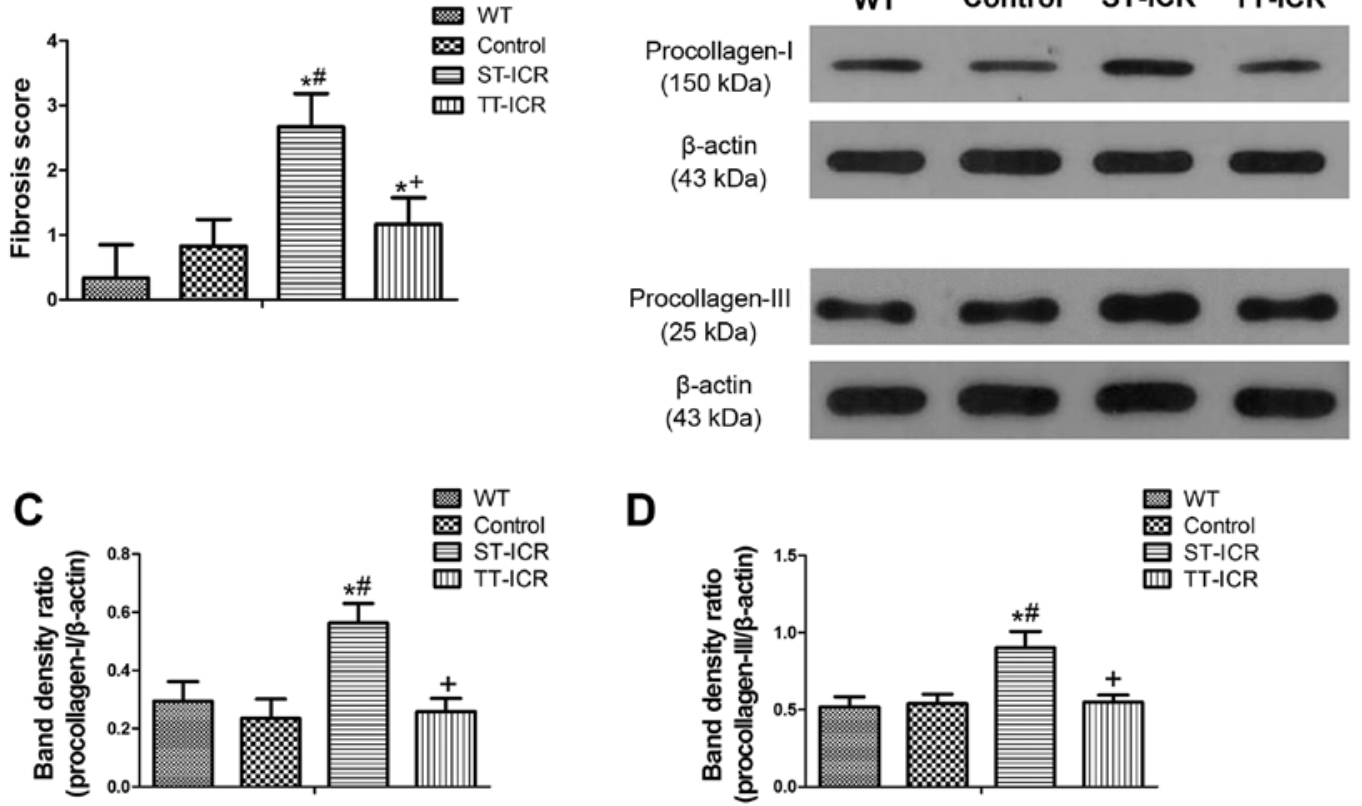

Figure 6. TPL attenuates anastomotic fibrosis and suppresses the synthesis of collagen. (A) Fibrosis score at anastomosis site in each group. (B) Results of western blot analysis for procollagen I and III in each group. The relative expression of procollagen I and III was statistically calculated compared to the $\beta$-actin level; (C) the ratios of the mean density of procollagen I at the anastomosis site in each group. (D) The ratios of the mean density of procollagen III at the anastomosis site in each group. The data are presented as the average $\pm \mathrm{SD}$ of 6 independent experiments. ${ }^{\prime \prime} \mathrm{P}<0.05$, significantly different from the $\mathrm{WT}$ group; ${ }^{\text {"}} \mathrm{P}<0.05$, significantly different from the control group; ${ }^{+} \mathrm{P}<0.05$, significantly different from the ST-ICR group. TPL, triptolide; WT, wild-type; ST-ICR, saline-treated-ICR group; TT-ICR, triptolide-treated ICR group.

A
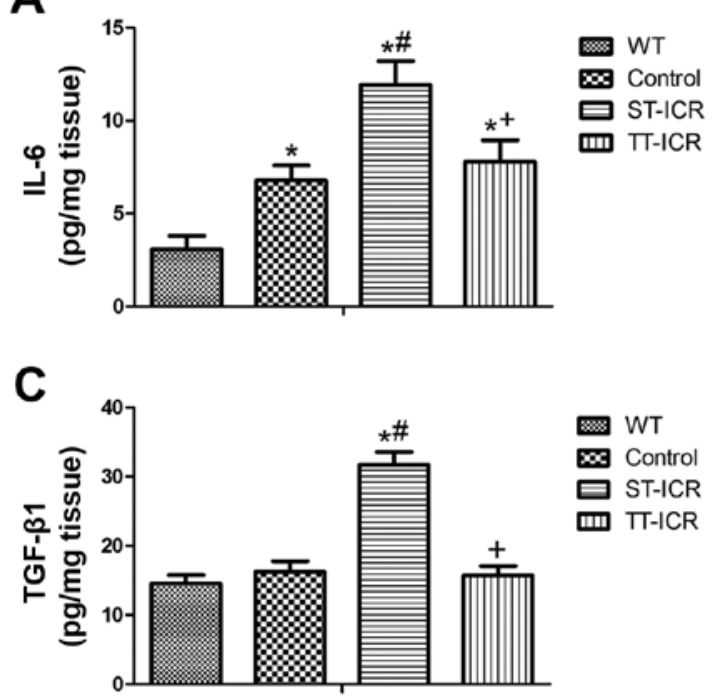

B

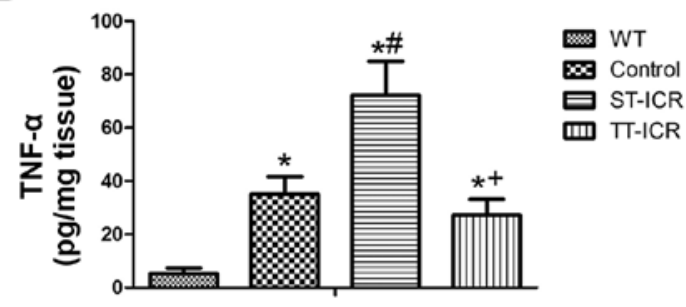

Figure 7. Average concentration of (A) IL-6, (B) TNF- $\alpha$ and (C) TGF- $\beta 1$ at the anastomosis site in each group. The data are presented as the average \pm SD of 6 independent experiments. ${ }^{*} \mathrm{P}<0.05$, significantly different from the WT group; ${ }^{*} \mathrm{P}<0.05$, significantly different from the control group; ${ }^{+} \mathrm{P}<0.05$, significantly different from the ST-ICR group. TNF- $\alpha$, tumor necrosis factor- $\alpha$; TGF- $\beta 1$, transforming growth factor- $\beta 1$; WT, wild-type; ST-ICR, saline-treated-ICR group; TT-ICR, triptolide-treated ICR group.

miR-16-1 (Fig. 8B-D). The level of HSP70 decreased along in the mice with anastomotic fibrosis severity at both the mRNA and protein level. Compared with the control group, the level of HSP70 in the ST-ICR group was significantly downregulated $(\mathrm{P}<0.05)$. However, a sharp rise in HSP70 expression was observed following treatment with TPL $(\mathrm{P}<0.05)$, which partly results from the inhibition of miR-16-1.

\section{Discussion}

$\mathrm{CD}$ is a chronic inflammatory gastrointestinal disorder that can affect any portion of the gastrointestinal tract, particularly the terminal ileum. Chronic and persistent inflammation induces intestinal fibrosis, and severe fibrosis can result in intestinal stenosis, which can lead to obstructions that require surgery 
A

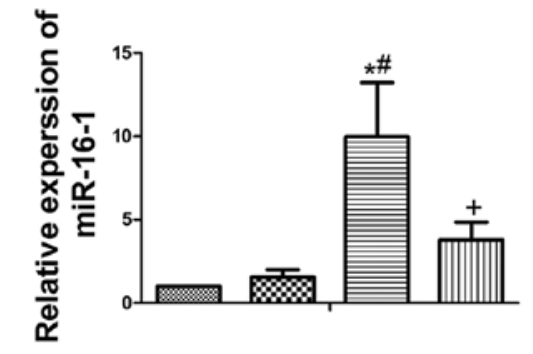

C

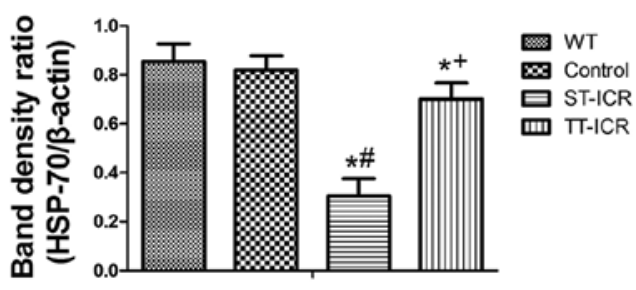

B

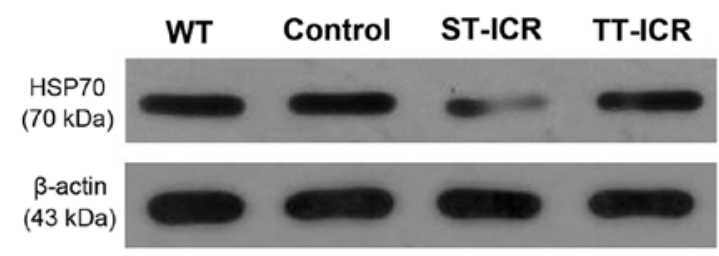

D

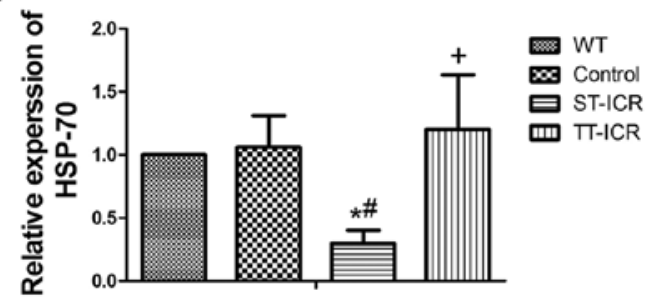

Figure 8. miR-16-1/HSP70 pathway is involved in anastomotic fibrosis in IL-10 ${ }^{-/}$mice undergoing ICR and can be regulated by TPL. (A) Relative expression of miR-16-1 at the anastomosis site in each group; (B) results of western blot analysis for HSP70 in each group. The relative expression of HSP70 was statistically calculated compared to the $\beta$-actin level; (C) the ratios of the mean density of HSP70 at the anastomosis site in each group. (D) Relative expression of HSP70 at the anastomosis site in each group; the data are presented as the average \pm SD of 6 independent experiments. * $P<0.05$, significantly different from the WT group; ${ }^{\#} \mathrm{P}<0.05$, significantly different from the control group; ${ }^{+} \mathrm{P}<0.05$, significantly different from the ST-ICR group. HSP70, heat shock protein 70 ; ICR, ileocecal resection; TPL, triptolide; WT, wild-type; ST-ICR, saline-treated-ICR group; TT-ICR, triptolide-treated ICR group.

or repeat surgery (21). The most frequent surgical strategy for patients with CD is ICR and ileum-colon anastomosis; however, patients undergoing ICR and re-anastomosis tend to have a higher relapse rate compared with patients with stricturoplasties or isolated small bowel resections. Recurrence frequently occurs around the anastomotic sites and the proximal intestine (22). The concrete mechanisms underlying postsurgical anastomotic fibrosis and stricture remain unknown. Therefore, further studies using animal models are warranted. Among numerous animal models, the IL-10 $0^{-/}$mouse model has features of Th1-/Th17-type inflammation similar to CD and has been extensively applied to study the pathogenesis of CD (23). As a novel model to study post-surgical pathological changes of anastomosis, the model of IL-10 $10^{-/}$mice undergoing ICR was constructed (7). In our study, no significant difference in either inflammation or fibrosis was detected between the WT and control groups; however, of note, the IL- 6 and TNF- $\alpha$ levels in the control group were significantly higher than those in the WT group. We hypothesize that this may result from the innate immune features of IL-10 $10^{-/-}$mice and environmental factors. IL-10 can inhibit the production of cytokines, including IFN- $\gamma$, IL-1, IL-6 and TNF- $\alpha$, from pro-inflammatory cells; therefore, this suppressive effect may be weakened by a lack of IL-10. However, intestinal inflammation and fibrosis in the IL-10 ${ }^{-/}$mice is intestinal-content dependent (particularly bacteria or bacterial products) (7); thus, the IL-10 $10^{-/}$mice used in our study, which were raised under SPF conditions, suffered only from local colitis and slight collagen accumulation. Therefore, we hypothesize that IL- $10^{-/-}$mice, which underwent ICR and were raised under SPF conditions, are appropriate models to investigate the pathogenesis of surgery-associated complications, particularly post-surgical anastomotic inflammation and fibrosis. In the present study, the upregulated inflammation and fibrosis score, the larger area of $\mathrm{CD}_{4}{ }^{+}$cell infiltration, as well as the overexpression of procollagen I and III in mice in the ST-ICR group, proved that the IL-10 ${ }^{-/-}$mice that underwent ICR, which have many features in common with $\mathrm{CD}$, can develop sustained and significant post-operative inflammation and anastomotic fibrosis. The pathogenesis can be attributed to mechanisms including impaired blood flow around the anastomosis site, an absence of the ileocecal valve and subsequent exposure of the anastomosis to luminal contents (7), or changes in the systemic immune response (22), as well as others. We showed that IL-10 $1{ }^{-/-}$mice that underwent ICR, models that precisely mimic post-surgical intestinal lesions of CD, provide an appropriate experimental platform with which to study the pathogenesis of inflammation and anastomotic fibrosis in patients with CD undergoing ICR.

The fibrotic process comprises a series of abnormal biological behaviors, including inflammatory cell infiltration, cytokine release, fibroblast differentiation and proliferation, and an imbalance in ECM synthesis and degradation. Previous studies have demonstrated the anti-inflammatory activity of TPL; however, an anti-fibrosis effect of this agent was still uncovered, and the therapeutic effects of TPL, attenuating fibrosis following intestinal anastomosis in this new model of CD have not been previously investigated, at least to the best of our knowledge. We demonstrated that following treatment with TPL, the inflammation and fibrosis levels at anastomosis sites of the mice in the TT-ICR groups were significantly attenuated compared with those in the ST-ICR group; additionally, TPL effectively inhibited the infiltration of $\mathrm{CD}_{4}^{+} \mathrm{T}$ cells and the synthesis of collagen at the anastomosis site. The elevated expression levels of TNF- $\alpha$, IL- 6 and TGF- $\beta 1$ were also reduced by TPL. As an effective risk-indicator of disease activity in CD, IL-6 can regulate $\mathrm{T}$ cell differentiation and activation, promote fibroblast proliferation and increase fibroblast collagen and tissue inhibitor of metalloproteinases-1 (TIMP-1) synthesis. TNF- $\alpha$ is alsoclosely 
related to the process of fibrosis. A previous study demonstrated that TNF- $\alpha$ found in chronic inflammatory conditions inhibits collagen phagocytosis and induces tissue fibrosis (12). Additionally, by reducing chemokine production by fibroblasts, TPL can also limit inflammatory cell infiltration (24). Matrix metalloproteinases (MMPs) are involved in the catabolism of ECM, and the activity of MMPs is restricted by TIMPs; imbalances between TIMPs and MMPs may result in disorders of ECM metabolism (25). TLRs, which are a family of pattern recognition receptors, represent receptors of the innate immune system. They activate downstream inflammatory responses and initiate the acquired immune response. The dysregulation of TLRs may induce chronic inflammation and tissue impairment in IBD. TPL can inhibit MMP expression and augment TIMP expression. Moreover, the level of TLR-2 and TLR-4 can also be reduced by TPL in the intestines of patients with CD (5). In mice with collagen-induced arthritis, TPL was found to inhibit IL-1 $\beta$, IL-6, TNF- $\alpha$, MMP-13 and MMP-3 expression and augment TIMP-1 and TIMP-2 expression, thereby suppressing the inflammatory reaction and preventing the development of the fibrotic process (26). In IL-1 $\beta$-treated human intervertebral disc cells, TPL significantly suppressed the expression of numerous genes, including IL-6, IL-8, MMP-1, MMP-2, MMP-3, MMP-13, TLR-2 and TLR-4 (27). TGF- $\beta$, which plays important roles in wound healing, the regulation of the immune system, metabolism of connective tissue, fibrosis and cancer progression, can drive fibrosis through multiple mechanisms, including the activation of the TGF- $\beta /$ Smad signaling pathway and epithelial to mesenchymal transition (EMT) (13). TPL can inhibit ECM protein synthesis by fibroblasts by suppressing Smad2 activation (28). A previous study found that TPL treatment markedly inhibited the expression of TGF- $\beta 1$ and attenuated cardiac fibrosis (29). TPL has also been reported to inhibit the TGF- $\beta$-induced phosphorylation of Smad 2 and Smad3, but to increase the level of Smad7 (30). Additionally, the NF- $\kappa \mathrm{B} / \mathrm{TNF}-\alpha / \mathrm{VCAM}-1$, TLR4-induced NF- $\kappa \mathrm{B} / \mathrm{IL}-1 \beta$ and TGF- $\beta 1 / \alpha-S M A / V i m e n t i n$ signaling pathways are all important targets of TPL (31). In particular, we have noticed that there are many miRNAs regulated by TPL, such as miR-344b-3p, miR-30b-3p (32) and miR-155 (33); as miR-16-1 is also an important target of TPL (17) and relates to the activity of CD, we hypothesized that the anti-fibrotic effects of TPL may be mediated partly through the inhibition of the expression of miR-16-1.

miRNAs are smallnon-coding RNA molecules thatcomprise approximately 22 nucleotides and function as inhibitors of gene expression by primarily binding to complementary sites on the 3'UTR of target mRNAs. miR-16-1, which was first reported to be associated with chronic lymphocytic lymphoma in 2002 (34), can regulate numerous cellular biological behaviors, including cell proliferation, differentiation, cell cycle regulation and apoptosis (35). Specifically, miR-16-1 has an increased expression both in the mucosa of the terminal ileum and in the peripheral blood of patients with active CD compared with healthy individuals $(15,16)$. The association between miR-16-1 and anastomotic fibrosis in patients with $\mathrm{CD}$ has not been previously investigated, at least to the best of our knowledge. A previous study showed that TPL can inhibit the expression of miR-16-1 in a time- and dose-dependent manner (17), which is in accordance with the findings of the present study. In our study, the expression of miR-16-1 was significantly inhibited by TPL, and the level of miR-16-1 positively correlated with the severity of inflammation and fibrosis in anastomosis. These results suggested that TPL may attenuate intestinal fibrosis partly through the downregulation of miR-16-1. Previous studies have tried to elucidate the association between miR-16-1 and inflammation and fibrosis. miR-16-1-expressing macrophages were found to promote the activation of purified $\mathrm{CD}_{4}{ }^{+} \mathrm{T}$ cells (36). A recent study demonstrated that $\mathrm{HCV}$ infection results in the overexpression of miR-16-1 and then inhibits the expression of Smad7 in the progression of liver fibrosis (37). Additionally, miR-16-1 is thought to be related to EMT, a significant contributor to the development of fibrosis (38). In addition, miR-16-1 functions through multiple mechanisms in a tissue- and cell-specific manner; for example, miR-16(-1) regulates $\mathrm{T}$ cell activation, proliferation and apoptosis by targeting Bcl2, CCND1, Erbb3, mTOR, Rictor and Runx1 (39-41). Recently, a study proved that miR-16-1 targets the 3'UTR of HSP70 and thus reduces HSP70 expression, and HSP70 was found to be crucial in the alleviation of intestinal damage $(42,43)$. Therefore, we further attempted to explore changes in HSP70 expression in this new model.

HSPs, which can be found in all eukaryotes and prokaryotes, are a family of highly conserved proteins that play important roles in cellular proliferation and differentiation and oncogenesis $(44,45)$. As the primary member of the HSP family, HSP70 has been shown to be involved in the pathogenesis of numerous chronic autoimmune diseases, including CD $(18,46)$. In the present study, the level of HSP70 decreased in mice with inflammation and fibrosis following ICR; however a sharp rise in HSP70 expression was observed following treatment with TPL, which partly results from the inhibition of miR-16-1. Besides, there is also a tight connection between HSP70 and anastomotic inflammation and fibrosis. Furthermore, the levels of IL-6, TGF- $\beta 1$ and TNF- $\alpha$ were all significantly elevated at the anastomosis site in the mice in the ST-ICR group and negatively correlated with the level of HSP70, and reversions were observed following treatment with TPL. As the TGF- $\beta$ / Smad pathway and EMT are all considered to be closely related to the level of TGF- $\beta$, the overexpression of HSP70 has been found to inhibit EMT by exerting domain-specific effects on Smad3 activation and nuclear translocation as well as increasing Smad7 expression in a dose-dependent manner (47). Additionally, HSP70 inhibits TGF- $\beta$ signal transduction by interacting with Smad2; the overexpression of HSP70 can inhibit the phosphorylation and nuclear translocation of Smad2 and blocks TGF- $\beta$-induced EMT (48). The overexpression of HSP70 has also been found to prevent the synthesis of cytokines, including IL- 6 and TNF- $\alpha$, and protects against TNF- $\alpha-$ and IL-6-induced intestinal damage $(42,43)$. Furthermore, HSP70 is involved in numerous signaling pathways, including nuclear factor- $\kappa \mathrm{B}$, Src, Akt and Raf (49). Through mechanisms such as regulating myofibroblast and $\mathrm{T}$ cell differentiation and inhibiting epithelial cell apoptosis, HSP70 is believed to effectively inhibit tissue inflammation and fibrosis $(18,19,49,50)$. We demonstrated that through the inhibition of the expression of miR-16-1, TPL significantly upregulated the HSP70 levels, and thus prevented the development of the fibrotic process in IL-10 ${ }^{-/-}$mice undergoing ICR. 
In conclusion, to the very best of our knowledge, the present study is the first to use the novel model of IL-10-deficient mice undergoing ICR to investigate the treatment utility of TPL on anastomotic fibrosis. The present study demonstrates that TPL is an effective substance against $\mathrm{CD}$ and exerts a protective role against post-surgical anastomotic fibrosis. The miR-16-1/ HSP70 signaling pathway, which is an important target of TPL, is a valuable therapeutic approach for $\mathrm{CD}$ that deserves further investigation.

\section{Acknowledgements}

This research was supported by funding from the National Natural Science Foundation of China (grant no. 81500421). We would sincerely like to thank Professor Qiu-Rong Li (Jinling Hospital), Dr Bao-Cai Wang (Technical University, Munich) and Professor Feng Gao (Medical School of Southeast University), as well as their team for their technical assistance.

\section{References}

1. Bernstein CN, Fried M, Krabshuis JH, Cohen H, Eliakim R, Fedail S, Gearry R, Goh KL, Hamid S, Khan AG, et al: World Gastroenterology Organization practice guidelines for the diagnosis and management of IBD in 2010. Inflamm Bowel Dis 16: 112-124, 2010.

2. Carter MJ, Lobo AJ and Travis SP; IBD Section, British Society of Gastroenterology: Guidelines for the management of inflammatory bowel disease in adults. Gut 53 (Suppl 5): V1-V16, 2004.

3. Ren J, Tao Q, Wang X, Wang Z and Li J: Efficacy of T2 in active Crohn's disease: A prospective study report. Dig Dis Sci 52: 1790-1797, 2007.

4. Titov DV, Gilman B, He QL, Bhat S, Low WK, Dang Y, Smeaton M, Demain AL, Miller PS, Kugel JF, et al: XPB, a subunit of TFIIH, is a target of the natural product triptolide. Nat Chem Biol 7: 182-188, 2011.

5. Yu C, Shan T, Feng A, Li Y,Zhu W, Xie Y,Li N and Li J: Triptolide ameliorates Crohn's colitis is associated with inhibition of TLRs/ NF- $\mathrm{BB}$ signaling pathway. Fitoterapia 82: 709-715, 2011.

6. Wei X, Gong J, Zhu J, Wang P, Li N, Zhu W and Li J: The suppressive effect of triptolide on chronic colitis and TNF-alpha/ TNFR2 signal pathway in interleukin-10 deficient mice. Clin Immunol 129: 211-218, 2008.

7. Rigby RJ, Hunt MR, Scull BP, Simmons JG, Speck KE, Helmrath MA and Lund PK: A new animal model of postsurgical bowel inflammation and fibrosis: The effect of commensal microflora. Gut 58: 1104-1112, 2009.

8. Murthy SN, Cooper HS, Shim H, Shah RS, Ibrahim SA and Sedergran DJ: Treatment of dextran sulfate sodium-induced murine colitis by intracolonic cyclosporin. Dig Dis Sci 38 : 1722-1734, 1993.

9. Berg DJ, Davidson N, Kühn R, Müller W, Menon S, Holland G, Thompson-Snipes L, Leach MW and Rennick D: Enterocolitis and colon cancer in interleukin-10-deficient mice are associated with aberrant cytokine production and CD4(+) TH1-like responses. J Clin Invest 98: 1010-1020, 1996.

10. Rath HC, Herfarth HH, Ikeda JS, Grenther WB, Hamm TE Jr, Balish E, Taurog JD, Hammer RE, Wilson KH and Sartor RB: Normal luminal bacteria, especially Bacteroides species, mediate chronic colitis, gastritis, and arthritis in HLA-B27/human beta2 microglobulin transgenic rats. J Clin Invest 98: 945-953, 1996.

11. Eckes B, Zigrino P, Kessler D, Holtkötter O, Shephard P, Mauch C and Krieg T: Fibroblast-matrix interactions in wound healing and fibrosis. Matrix Biol 19: 325-332, 2000.

12. Chou DH, Lee W and McCulloch CA: TNF-alpha inactivation of collagen receptors: Implications for fibroblast function and fibrosis. J Immunol 156: 4354-4362, 1996.

13. Morikawa M, Derynck R and Miyazono K: TGF- $\beta$ and the TGF- $\beta$ family: Context-dependent roles in cell and tissue physiology. Cold Spring Harb Perspect Biol 8: 8, 2016.

14. Lochhead P, Khalili H, Ananthakrishnan AN, Richter JM and Chan AT: Association between circulating levels of c-reactive protein and interleukin- 6 and risk of inflammatory bowel disease. Clin Gastroenterol Hepatol 14: 818-824, 2016.
15. Iborra M, Bernuzzi F, Correale C, Vetrano S, Fiorino G, Beltrán B, Marabita F, Locati M, Spinelli A, Nos P, et al: Identification of serum and tissue micro-RNA expression profiles in different stages of inflammatory bowel disease. Clin Exp Immunol 173: 250-258, 2013.

16. Paraskevi A, Theodoropoulos G, Papaconstantinou I, Mantzaris G, Nikiteas N and Gazouli M: Circulating MicroRNA in inflammatory bowel disease. J Crohn's Colitis 6: 900-904, 2012.

17. Meng HT, Zhu L, Ni WM, You LS, Jin J and Qian WB: Triptolide inhibits the proliferation of cells from lymphocytic leukemic cell lines in association with downregulation of NF- $\mathrm{\kappa B}$ activity and miR-16-1*. Acta Pharmacol Sin 32: 503-511, 2011.

18. Bellaye PS, Burgy O, Causse S, Garrido C and Bonniaud P: Heat shock proteins in fibrosis and wound healing: Good or evil? Pharmacol Ther 143: 119-132, 2014.

19. Hauet-Broere F, Wieten L, Guichelaar T, Berlo S, van der Zee R and Van Eden W: Heat shock proteins induce T cell regulation of chronic inflammation. Ann Rheum Dis 65 (Suppl 3): iii65-iii68, 2006.

20. Zhang $\mathrm{Z}$ and Cheng Y: miR-16-1 promotes the aberrant $\alpha$-synuclein accumulation in parkinson disease via targeting heat shock protein 70. ScientificWorldJournal 2014: 938348, 2014.

21. Li C and Kuemmerle JF: Mechanisms that mediate the development of fibrosis in patients with Crohn's disease. Inflamm Bowel Dis 20: 1250-1258, 2014.

22. Borowiec AM, Sydora BC, Doyle J, Guan LL, Churchill TA, Madsen K and Fedorak RN: Small bowel fibrosis and systemic inflammatory response after ileocolonic anastomosis in IL-10 null mice. J Surg Res 178: 147-154, 2012.

23. Kühn R, Löhler J, Rennick D, Rajewsky K and Müller W: Interleukin-10-deficient mice develop chronic enterocolitis. Cell 75: 263-274, 1993.

24. Liu Y,Li J,Liu Y, Wang P and Jia H: Inhibition of zymosan-induced cytokine and chemokine expression in human corneal fibroblasts by triptolide. Int J Ophthalmol 9: 9-14, 2016.

25. Burger D, Rezzonico R, Li JM, Modoux C, Pierce RA, Welgus HG and Dayer JM: Imbalance between interstitial collagenase and tissue inhibitor of metalloproteinases 1 in synoviocytes and fibroblasts upon direct contact with stimulated T lymphocytes: Involvement of membrane-associated cytokines. Arthritis Rheum 41: 1748-1759, 1998.

26. Lin N, Liu C, Xiao C, Jia H, Imada K, Wu H and Ito A: Triptolide, a diterpenoid triepoxide, suppresses inflammation and cartilage destruction in collagen-induced arthritis mice. Biochem Pharmacol 73: 136-146, 2007.

27. Klawitter M, Quero L, Klasen J, Liebscher T, Nerlich A, Boos N and Wuertz K: Triptolide exhibits anti-inflammatory, anti-catabolic as well as anabolic effects and suppresses TLR expression and MAPK activity in IL-1 $\beta$ treated human intervertebral disc cells. Eur Spine J 21 (Suppl 6): S850-S859, 2012.

28. Zhu B, Wang YJ, Zhu CF, Lin Y, Zhu XL, Wei S, Lu Y and Cheng XX: Triptolide inhibits extracellular matrix protein synthesis by suppressing the Smad 2 but not the MAPK pathway in TGF-beta1-stimulated NRK-49F cells. Nephrol Dial Transplant 25: 3180-3191, 2010.

29. Zhang Z, Qu X, Ni Y, Zhang K, Dong Z, Yan X, Qin J, Sun H, Ding Y, Zhao $\mathrm{P}$, et al: Triptolide protects rat heart against pressure overload-induced cardiac fibrosis. Int J Cardiol 168 : 2498-2505, 2013

30. Chen M, Lv Z, Huang L, Zhang W, Lin X, Shi J, Zhang W, Liang R and Jiang S: Triptolide inhibits TGF- $\beta 1$-induced cell proliferation in rat airway smooth muscle cells by suppressing Smad signaling. Exp Cell Res 331: 362-368, 2015.

31. Guo X, Xue M, Li CJ, Yang W, Wang S, Ma Z, Zhang X, Wang X, Zhao R, Chang B and Chen LM: Protective effects of triptolide on TLR4 mediated autoimmune and inflammatory response induced myocardial fibrosis in diabetic cardiomyopathy. J Ethnopharmacol 193: 333-344, 2016.

32. Jiang CB, Wei MG, Tu Y, Zhu H, Li CQ, Jing WM and Sun W: Triptolide attenuates podocyte injury by regulating expression of miRNA-344b-3p and miRNA-30b-3p in rats with adriamycin-induced nephropathy. Evidence-based complementary and alternative medicine : eCAM 2015: 107814, 2015. https://doi. org/10.1155/2015/107814

33. Wu R, Li Y, Guo Z, Gong J, Zhu W, Li N and Li J: Triptolide ameliorates ileocolonic anastomosis inflammation in IL-10 deficient mice by mechanism involving suppression of miR-155/ SHIP-1 signaling pathway. Mol Immunol 56: 340-346, 2013. 
34. Calin GA, Dumitru CD, Shimizu M, Bichi R, Zupo S, Noch E, Aldler H, Rattan S, Keating M, Rai K, et al: Frequent deletions and down-regulation of micro- RNA genes miR15 and miR16 at $13 q 14$ in chronic lymphocytic leukemia. Proc Natl Acad Sci USA 99: 15524-15529, 2002.

35. Li F, Xu Y, Deng S, Li Z, Zou D, Yi S, Sui W, Hao M and Qiu L: MicroRNA-15a/16-1 cluster located at chromosome $13 \mathrm{q} 14$ is downregulated but displays different expression pattern and prognostic significance in multiple myeloma. Oncotarget 6: 38270-38282, 2015.

36. Jia X, Li X, Shen Y, Miao J, Liu H, Li G and Wang Z: MiR-16 regulates mouse peritoneal macrophage polarization and affects T-cell activation. J Cell Mol Med 20: 1898-1907, 2016.

37. Zhu B, Wei XX, Wang TB, Zhou YC, Liu AM and Zhang GW: Increased miR-16 expression induced by hepatitis $C$ virus infection promotes liver fibrosis through downregulation of hepatocyte growth factor and Smad7. Arch Virol 160: 2043-2050, 2015.

38. Shi L, Jackstadt R, Siemens H, Li H, Kirchner T and Hermeking H: p53-induced miR-15a/16-1 and AP4 form a double-negative feedback loop to regulate epithelial-mesenchymal transition and metastasis in colorectal cancer. Cancer Res 74: 532-542, 2014.

39. Liu L, Walker EA, Kissane S, Khan I, Murray PI, Rauz S and Wallace GR: Gene expression and miR profiles of human corneal fibroblasts in response to dexamethasone. Invest Ophthalmol Vis Sci 52: 7282-7288, 2011.

40. Marcais A, Blevins R, Graumann J, Feytout A, Dharmalingam G, Carroll T, Amado IF, Bruno L, Lee $\mathrm{K}$, Walzer T, et al: microRNA-mediated regulation of mTOR complex components facilitates discrimination between activation and anergy in CD4 T cells. J Exp Med 211: 2281-2295, 2014.

41. Rouse M, Rao R, Nagarkatti M and Nagarkatti PS: 3,3'-diindolylmethane ameliorates experimental autoimmune encephalomyelitis by promoting cell cycle arrest and apoptosis in activated T cells through microRNA signaling pathways. J Pharmacol Exp Ther 350: 341-352, 2014.
42. Van Molle W, Wielockx B, Mahieu T, Takada M, Taniguchi T, Sekikawa K and Libert C: HSP70 protects against TNF-induced lethal inflammatory shock. Immunity 16: 685-695, 2002.

43. Meng K, Liu Q, Dou Y and Huang Q: Prior peritoneal lavage with hot $0.9 \%$ saline induces HSP70 expression and protects against cerulein-induced acute pancreatitis in rats. Mol Biol Rep 40: 1443-1449, 2013.

44. Sherman MY and Gabai VL: Hsp70 in cancer: Back to the future. Oncogene 34: 4153-4161, 2015.

45. Murphy ME: The HSP70 family and cancer. Carcinogenesis 34: 1181-1188, 2013

46. Abou El Azm AR, Yousef M, Kobtan A, Awad A, Elkassas G and Elfert A: Colonic mucosal expression of heat-shock proteins may have a potential prognostic value in ulcerative colitis. Arab journal of gastroenterology: the official publication of the Pan-Arab Association of Gastroenterology 16: 20-24, 2015. https://doi.org/10.1016/j.ajg.2015.02.005.

47. Zhou Y, Mao H, Li S, Cao S, Li Z, Zhuang S, Fan J, Dong X, Borkan SC, Wang Y, et al: HSP72 inhibits Smad3 activation and nuclear translocation in renal epithelial-to-mesenchymal transition. J Am Soc Nephrol 21: 598-609, 2010.

48. Li Y, Kang X and Wang Q: HSP70 decreases receptor-dependent phosphorylation of Smad2 and blocks TGF-beta-induced epithelial-mesenchymal transition. Journal of genetics and genomics = Yi chuan xue bao 38: 111-116, 2011.

49. Tao Y, Hart J, Lichtenstein L, Joseph LJ, Ciancio MJ, Hu S, Chang EB and Bissonnette M: Inducible heat shock protein 70 prevents multifocal flat dysplastic lesions and invasive tumors in an inflammatory model of colon cancer. Carcinogenesis 30: 175-182, 2009.

50. Mao H, Li Z, Zhou Y, Li Z, Zhuang S, An X, Zhang B, Chen W, Nie J, Wang Z, et al: HSP72 attenuates renal tubular cell apoptosis and interstitial fibrosis in obstructive nephropathy. Am J Physiol Renal Physiol 295: F202-F214, 2008. 